TuR1

$1: 30 \mathrm{pm}-1: 45 \mathrm{pm}$

\title{
Experimental characterization of dispersion properties of the leaky modes in planar photonic crystal waveguide
}

\author{
Marko Lončar, California Institute of Technology, MS 136-93, Pasadena, CA 91125 \\ Dušan Nedeljković, Thomas P. Pearsall, CERF, Corning, SA 77210 Avon, France \\ Jelena Vučković, Axel Scherer, California Institute of Technology, MS 136-93, Pasadena, CA 91125 \\ Sergey Kuchinsky, Corning Scientific Center, 199034 St. Petersburg, Russia \\ Douglas C. Allan, SP-FR-05, Corning Incorporated, Corning, NY 14831 \\ e-mail: loncar@caltech.edu, phone: (626) 395-2207, fax: (626) 683-9547, http://nanofab.caltech.edu
}

The planar photonic crystal structure (PPC) that we are interested in is a silicon slab, suspended in air and perforated with a 2D triangular lattice of holes with radius $r=0.4 a$, where $a=530 \mathrm{~nm}$ is the periodicity of the lattice. The thickness of the slab is $t=300 \mathrm{~nm}$. The waveguide is defined as a row of missing holes. In this work we have characterized waveguides with discontinuities in the form of a single defect cavity, as shown in Figure 1. Details on fabrication procedure can be found in our previous publication'.

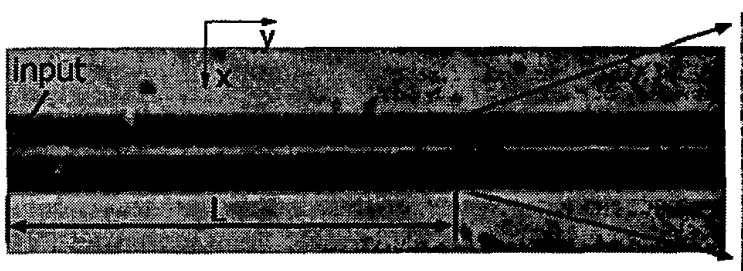

Figure 1. PPC waveguide with discontinuity in the form of single defect cavity.
Butt-coupling of a single-mode fiber was used to introduce light from a tunable semiconductor diode laser into the PPC waveguide. Waveguiding performance was observed by visualization of the guiding structure with infrared camera positioned in the plane perpendicular to the sample. This camera was used to detect the light scattered in the vertical direction (out of plane loss). In our previous work ${ }^{2}$ we have used similar setup to analyze waveguides with sharp bends, and we have observed significant out of plane losses when coupling was not optimized. We have attributed those losses to the leaky modes of the waveguide. In this work we investigate this phenomenon in more detail.

In Figure 2 we show signal detected with camera for different wavelengths of the input light.
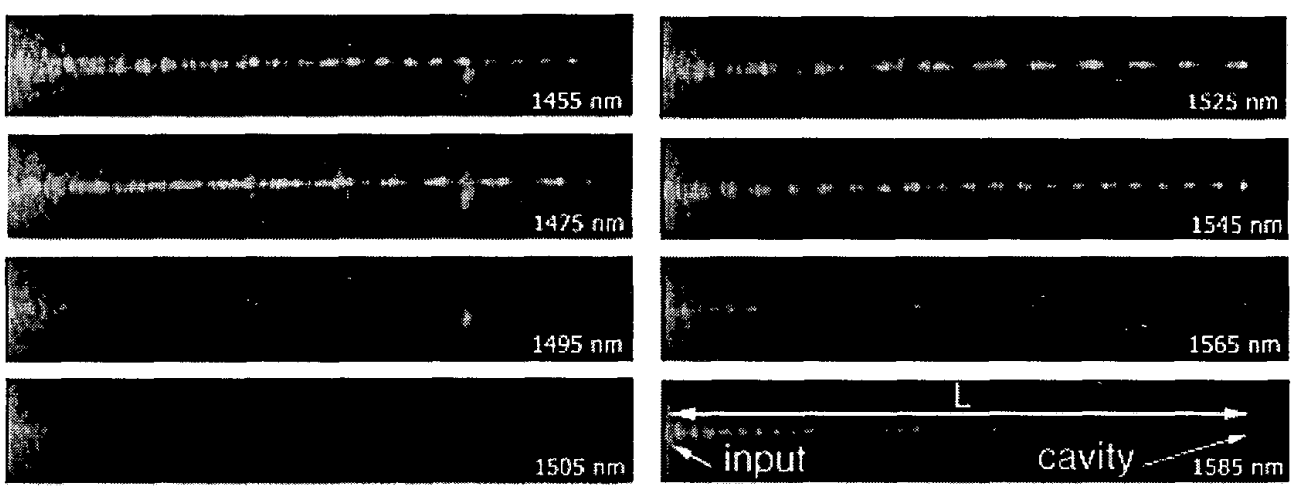

Figure 2. Wavelength dependence of the signal detected by the camera. Periodic modulation pattern can be observed.

At nearly every wavelength, a clear periodic intensity modulation can be seen along the waveguide direction. Periodicity of this modulation grows shorter as the difference between the wavelength and $1500 \mathrm{~nm}$ grows larger, in either direction of the wavelength. However, for wavelengths in the range $(1495 \mathrm{~nm}, 1505 \mathrm{~nm})$ this modulation intensity has nearly disappeared. It is important to say that the modulation pattern shown in Figure 2 was detected above the sample surface, that is at different focal distance from the one used to image the surface of the sample (Figure 1). We have used the fast Fourier transform (FFT) to extract the information on the spatial periodicity of the 


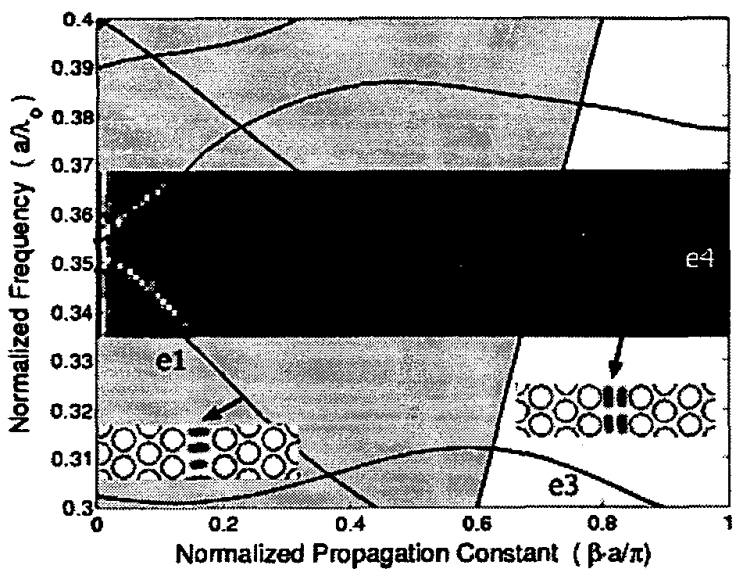

Figure 3. Calculated (black solid lines) and experimentally obtained (white dots in dark gray region) dispersion diagram for the TE-like modes of the PPC waveguide. Mode profiles for some modes are also shown.

modulation pattern, for each normalized frequency $a / \lambda$. The results of the FFT analysis of the experimental results are overlaid onto calculated theoretical results and are presented in Figure 3. The black solid lines in this figure represent results from our 3D FDTD simulation. The region shown in dark gray color is the result of the FFT - the normalized propagation constants detected from the experimental results are represented by white dots for each normalized frequency. It can be seen that our experimental results are in a very good agreement with 3D FDTD simulations. Experimental results show the presence of a small stop-band around $a / \lambda$ $=0.353$, as predicted by 3D FDTD analysis. This mini stop band corresponds to the wavelength range $(1495 \mathrm{~nm}, 1505 \mathrm{~nm})$ in which no periodic modulation pattern was observed (Figure 2). Therefore, we can conclude that the periodic modulation pattern detected by the camera is due to coupling of light from the external light source into the leaky modes of the waveguide.

In order to check this hypothesis even further we have modeled, using 3D FDTD code, waveguide sections closed with PPC at both sides. This is similar to the situation that we have in the experiment where waveguide sections are closed with cleaved facet at one side and single defect cavity at the other side. In our experiment cavity acts like mirror since its eigen-mode frequency $(a / \lambda=0.326)$ is outside the frequency range probed in the experiment (dark gray region in Figure 2$)$ and therefore light in the waveguide cannot couple to the cavity mode. Due to memory limitations of the computers used in the

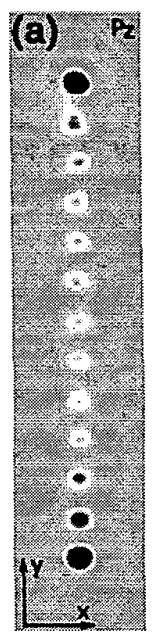

$\mathrm{a} / \lambda=0.31$
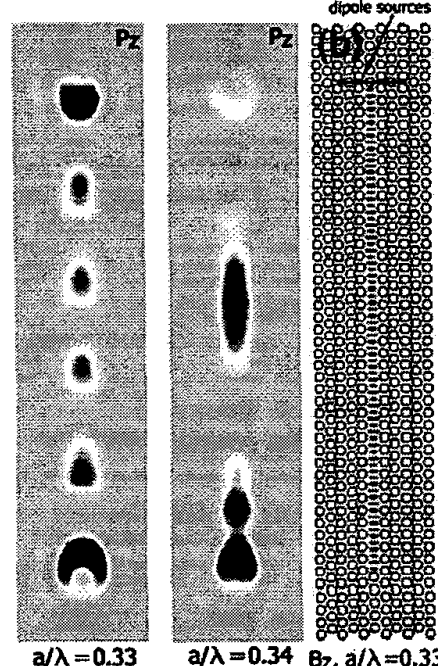

Figure 4. (a) Frequency dependence of the $P_{z}$ component of the Pointing vector, result of $3 D$ FDTD analysis. Periodic modulation pattern is formed above the surface of the sample. (b) Mode profile at the middle of the slab for $\mathrm{a} / \lambda=0.33$. simulations, modeled waveguide sections were about 6 times smaller than the actual structures tested in the experiments. The waveguide sections were excited with dipole sources of fixed frequency.

In Figure 4(a) we show the $\mathrm{P}_{\mathrm{z}}$ components of the Pointing vector at about $1 \mu \mathrm{m}$ above the sample surface, for three different normalized frequencies. Clear periodic modulation pattern, very similar to the one observed in the experiment (Figure 2), can be seen. The spatial periodicity of the modulation pattern shows the same dependence on the frequency of light as in the experiment - for frequencies below the mini stop band, spatial periodicity grows larger as the frequency increases. Moreover, the spatial profile of the mode excited in the waveguide [Figure 4(b)] has the same symmetry as the leaky el mode (Figure 3 ), thus confirming our hypothesis of coupling the light into the leaky modes of the structure.

In conclusion, we have experimentally obtained the dispersion diagram of the leaky modes in the planar photonic crystal waveguide for the wavelengths from $1440 \mathrm{~nm}$ to 1590 nm. A small stop band, around $\lambda=1500 \mathrm{~nm}$ is also detected. The experimentally obtained results are in very good agreement with our 3D FDTD calculations.

[1] M. Lončar, T. Doll, J. Vučković, A. Scherer, J. of

Lightwave Tech., 18, 1402 (2000)

[2] M. Lončar, D. Nedeljković, T. Doll, J. Vučković, A. Scherer, T. P. Pearsall, Appl. Phys. Lett., 77, 1937 (2000) 